\begin{tabular}{|c|c|}
\hline Title & $\begin{array}{l}\text { Meaning of the Rational Solution Obtained by Game Theory in a Multi-Objective Electromagnetic A pparatus Design } \\
\text { Problem }\end{array}$ \\
\hline Author(s) & Noguchi, So; Miyamoto, Tomoyuki; Matsutomo, Shinya \\
\hline Citation & $\begin{array}{l}\text { IEEE Transactions on Magnetics, 50(2), } 7016004 \\
\text { https://doi.org/10.1109// MA G.2013.2281848 }\end{array}$ \\
\hline Issue Date & 201402 \\
\hline Doc URL & http:/hdl.handle.net/2115/56402 \\
\hline Rights & $\begin{array}{l}\text { C } 2014 \text { IEEE. Personal use of this material is permitted. Permission from IEEE must be obtained for all other uses, in } \\
\text { any current or future media, including reprinting/republishing this material for advertising or promotional purposes, } \\
\text { creating new collective works, for resale or redistribution to servers or lists, or reuse of any copyrighted component of } \\
\text { this work in other works. }\end{array}$ \\
\hline Tyре & article (author version) \\
\hline File Information & GameTheory_final.pdf \\
\hline
\end{tabular}

Instructions for use 


\title{
Meaning of the Rational Solution Obtained by Game Theory in Multiobjective Electromagnetic Apparatus Design Problem
}

\author{
So Noguchi ${ }^{1}$, Tomoyuki Miyamoto ${ }^{1}$, and Shinya Matsutomo ${ }^{2}$ \\ ${ }^{1}$ Graduate School of Information Science and Technology, Hokkaido University, Sapporo 060-0814, Japan \\ ${ }^{2}$ Department of Electronic Control Engineering, Niihama National College of Technology, Niihama 792-8580, Japan
}

\begin{abstract}
The optimization method is often used for designing the configuration of electromagnetic apparatuses, in order to enhance the performance. Many optimal design methods have been developed and popularized. However, most of the optimal design methods can take into account a single objective function. Therefore, when the problem has a few purposes, a weighted summed objective function is usually employed. Recently, we have proposed a new method taking into account the multiple purposes simultaneously and individually. The proposed method can select one rational solution from among the Pareto optima, based on the game theory. However, the meaning of the selected rational solution is unclear. Therefore, the meaning is investigated by calculating the weight parameters of the weighted summed objective function so that the optimal solution of the weighted summed objective function is identical to the rational solution of the game theory. We can intuit the meaning of the rational solution by comparing with the weight parameters.
\end{abstract}

Index Terms - Game theory, multi-objective optimization, optimal design, Pareto optimum.

\section{INTRODUCTION}

$\mathbf{R}$ ECENTLY, an optimal design method consisting of a numerical simulation method and an optimization technique is often used for the design of electromagnet apparatuses, such as motors, generators, and transformers [1]-[3], for the sake of saving time, cost, and labor. For popularizing the optimal design method more, it is necessary to optimize multiple objectives simultaneously and individually. Most of the common optimization methods, such as genetic algorithm, simulated annealing, and so on, can optimize a single objective function. Hence, the weighted summed objective function is often employed for taking into account the multiple objectives. However, the multiple objectives often present conflicting and trade-off characteristic, and the optimal design problem with multiple objectives builds a Pareto front. Many methods of specifying the Pareto front have been proposed [4], [5]. It is difficult to select a single solution worthy of the optimal design from among the Pareto optimal solutions. In fact, the weight parameters are decided empirically, and it is hard to comprehend the meaning of the weight parameters decided.

We have previously proposed a multi-purposed optimal design method utilizing the game theory [6]. The proposed method can rationally select one solution worthy of the design from among the Pareto optima. It is unnecessary to decide the weight parameters in the method. A surface permanent magnet (SPM) motor was rationally optimized by the proposed method with three objectives of minimizing the copper and iron losses and the cogging torque. It was successful to minimize the multiple objectives without the weight parameters, however the meaning of the obtained rational solution was intuitively unclear. Therefore, in this paper, the weight parameters of the weighted summed objective function are specified from the

Manuscript received June 29, 2013. Corresponding author: S. Noguchi (email: noguchi@ssi.ist.hokudai.ac.jp). obtained rational solution. By comparing the worth of each weight parameter, the meaning of the rational solution will be clarified.

\section{Optimal Design of SPM Motor Based on Game THEORY}

When electromagnetic apparatuses with more than two conflicting objectives are optimally designed, the candidates of the optimal solution exist on the Pareto front (see Fig. 1). The optimal solution to be selected has to be on the Pareto front usually. The game theory [7], [8] was utilized in order to select a rational solution [6], [9].

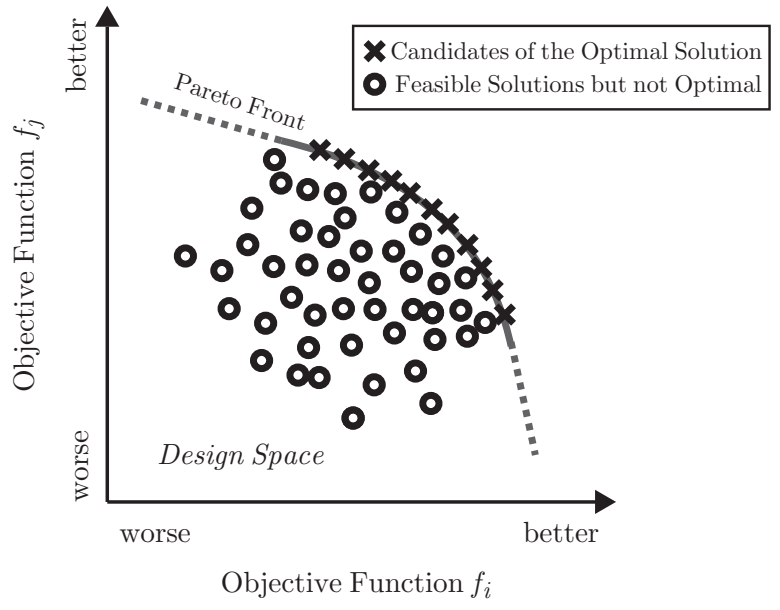

Fig. 1. An optimization method explores an optimal solution in the feasible solutions (circles and crosses). The candidates of the optimal solution (crosses) usually exist on the Pareto front. It is, however, hard to select one optimal solution from among the candidates of the Pareto optima.

The SPM motor was optimized by the method based on the game theory [6]. Fig. 2 shows the configuration of the SPM 
motor with the design parameters, and Table I shows the specifications of the SPM motor [10]. In [6], the three objective functions $f$ were simultaneously maximized as follows:

$$
\begin{aligned}
& f_{1}=100 / W_{\mathrm{c}} \\
& f_{2}=100 / W_{\mathrm{i}} \\
& f_{3}=1 / T_{\mathrm{c}}
\end{aligned}
$$

where $W_{\mathrm{c}}, W_{\mathrm{i}}$, and $T_{\mathrm{c}}$ are the copper and the iron loss, and the cogging torque, respectively. The design variables $x_{2}, x_{4}$, and $x_{6}$ were optimized by the game theory, whereas the other variables were optimized by the simple genetic algorithm. Table II shows the optimized design variables.

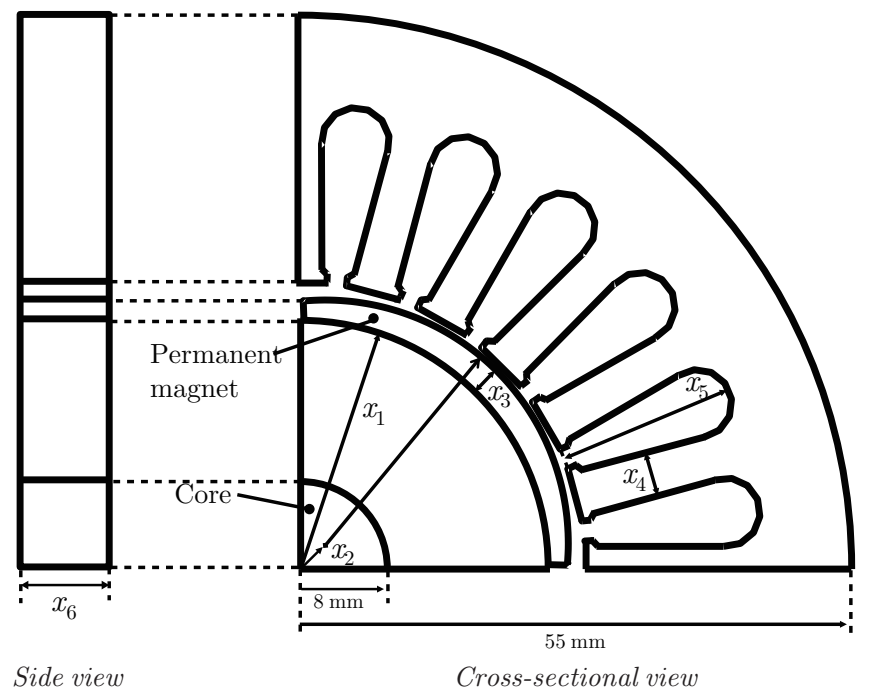

Fig. 2. The configuration of the SPM motor to be optimally designed with the six design variables. The other two design variables are the wire diameter $x_{7}$ and the phase of supply voltage $x_{8}$. The variables $x_{2}, x_{4}$, and $x_{6}$ are optimized by the game theory.

TABLE I

SPECIFICATIONS OF SPM MOTOR

\begin{tabular}{lr} 
Rated torque & $1.7 \mathrm{Nm}$ \\
Rated speed & $4000 \mathrm{rpm}$ \\
Residual magnetic flux density of permanent magnet & $1.2 \mathrm{~T}$ \\
Space factor of wire in a slot & less than $60 \%$ \\
\hline
\end{tabular}

TABLE II

OPTIMIZED DESIGN VARIABLES

\begin{tabular}{l|rr}
\hline & Variable & Optimized value \\
\hline Radius of rotor core & $x_{1}(\mathrm{~mm})$ & 50.0 \\
Center position for outer arc & $x_{2}(\mathrm{~mm})$ & 3.5 \\
surface of permanent magnet & & \\
Permanent magnet thickness & $x_{3}(\mathrm{~mm})$ & 1.95 \\
Teeth width & $x_{4}(\mathrm{~mm})$ & 2.5 \\
Teeth height & $x_{5}(\mathrm{~mm})$ & 20.0 \\
Motor thickness & $x_{6}(\mathrm{~mm})$ & 50.0 \\
Wire diameter & $x_{7}(\mathrm{~mm})$ & 1.25 \\
Supply voltage phase & $x_{8}(\mathrm{deg})$. & 13.0 \\
\hline
\end{tabular}

Every design variable is individually related to a single objective in the game theory. The first objective function (1) related to the copper loss is dominated by the motor thickness $x_{6}$, the second one (2) related to the iron loss is dominated by the teeth width $x_{4}$, and the third one (3) related to the cogging torque is dominated by the center position of the outer arc surface of permanent magnet $x_{2}$.

\section{Meaning of the Rational Solution}

The rational solution can be obtained by the game theory [6], however its meaning is unclear. In order to clarify the meaning, the weight parameters of the weighted summed objective function are specified so that the optimal solution of the weighted summed objective function is identical to that of the game theory. The way of specifying the weight parameters are described below.

Firstly, the weighted summed objective function $F$ to be minimized is defined as

$$
F=\sum_{i=1}^{n} w_{i} \frac{1}{f_{i}}
$$

where $w, f$, and $n$ are the weight parameter of the weighted summed objective function, the single objective function, and the number of the objectives, respectively. Additionally, the weight parameters are conditioned as follows:

$$
\sum_{i=1}^{n} w_{i}=1
$$

Here, it is assumed that the Pareto front is convex locally around the rational solution obtained by the game theory. When the value of two arbitrary objective functions are $\left(\overline{f_{i}}, \overline{f_{j}}\right)$ at the rational solution, there is a tangent line touching the Pareto front at the point $\left(\overline{f_{i}}, \overline{f_{j}}\right)$, as shown in Fig. 3. Here, the tangent line is represented by

$$
f_{j}=a_{i j} f_{i}+b_{i j}
$$

where $a_{i j}$ and $b_{i j}$ are the constant parameters. Considering only two arbitrary objectives, the optimization problem is transformed to the minimization of

$$
F_{i j}=w_{i} \frac{1}{f_{i}}+w_{j} \frac{1}{f_{j}} .
$$

Since the rational solution is on the Pareto front, (6) is substituted into (7). The differential of $F_{i j}$ becomes 0 for minimizing $F_{i j}$, as follows:

$$
\frac{\mathrm{d} F_{i j}}{\mathrm{~d} f_{i}}=-\frac{w_{i}}{f_{i}^{2}}-\frac{a_{i j} w_{j}}{f_{j}^{2}}=0 .
$$

Solving (8) with (5), the weight parameters $\overline{w_{i}}(i=1, \cdots, n)$ are decided.

When the weighted summed objective function $F$ of (4) with $\overline{w_{i}}$ are minimized, its solution must correspond to the rational solution. That is, the meaning of the rational solution will be clarified from the obtained weight parameters $\overline{w_{i}}$. 


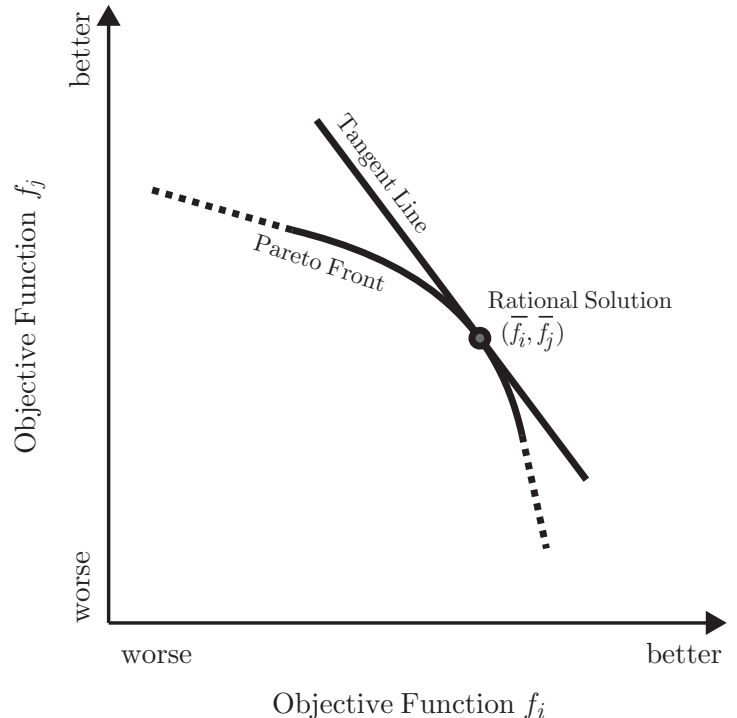

Fig. 3. The relation of the Pareto front and the tangent line. The Pareto front touches the tangent line at the rational solution which is obtained by the game theory.

\section{Meaning of the Rational Solution of Optimal SPM MOTOR DESIGN}

The aforementioned optimal design problem of the SPM has the three objective functions $f_{1}, f_{2}$, and $f_{3}$. The objective function values (rational solution) obtained by the game theory are

$$
\begin{aligned}
& \overline{f_{1}}=2.89 \\
& \overline{f_{2}}=11.89, \\
& \overline{f_{3}}=37.91
\end{aligned}
$$

with $x_{6}=50.0, x_{4}=2.5$, and $x_{2}=3.5$ [6]. Fig. 4 shows the optimized configuration of the SPM motor utilizing the game theory.

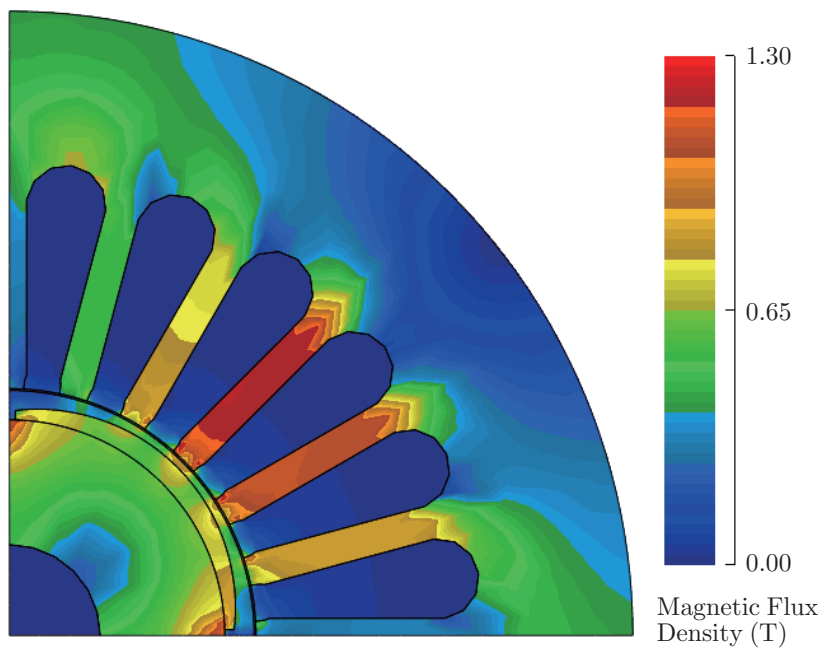

Fig. 4. The configuration of the SPM motor, which is optimized by the game theory [6].
Two local objective functions are derived from (7):

$$
\begin{aligned}
& F_{31}=w_{3} \frac{1}{f_{3}}+w_{1} \frac{1}{f_{1}} \\
& F_{32}=w_{3} \frac{1}{f_{3}}+w_{2} \frac{1}{f_{2}} .
\end{aligned}
$$

The differential functions are

$$
\begin{aligned}
\frac{\mathrm{d} F_{31}}{\mathrm{~d} f_{3}} & =-\frac{w_{3}}{f_{3}^{2}}-\frac{a_{31} w_{1}}{f_{1}^{2}}=0 \\
\frac{\mathrm{d} F_{32}}{\mathrm{~d} f_{3}} & =-\frac{w_{3}}{f_{3}^{2}}-\frac{a_{32} w_{2}}{f_{2}^{2}}=0
\end{aligned}
$$

where the tangent lines are defined as

$$
\begin{aligned}
& f_{1}=a_{31} f_{3}+b_{31} \\
& f_{2}=a_{32} f_{3}+b_{32} .
\end{aligned}
$$

Solving (14) and (15) with (5), the weight parameters are obtained as follows:

$$
\begin{aligned}
w_{1} & =\frac{a_{32} f_{1}^{2}}{a_{32} f_{1}^{2}+a_{31} f_{2}^{2}-a_{31} a_{32} f_{3}^{2}} \\
w_{2} & =\frac{a_{31} f_{2}^{2}}{a_{32} f_{1}^{2}+a_{31} f_{2}^{2}-a_{31} a_{32} f_{3}^{2}} \\
w_{3} & =-\frac{a_{31} a_{32} f_{3}^{2}}{a_{32} f_{1}^{2}+a_{31} f_{2}^{2}-a_{31} a_{32} f_{3}^{2}} .
\end{aligned}
$$

Figs. 5 and 6 show the plot of the search points in the objective functions $f_{1}$ vs. $f_{3}$ and $f_{2}$ vs. $f_{3}$, respectively. The rational solution of the SPM motor design problem exists on the Pareto front. The tangent lines, as shown in Figs. 5 and 6, are obtained as

$$
\begin{aligned}
& f_{1}=-1.250 f_{3}+41.532 \\
& f_{2}=-2.425 f_{3}+67.624 .
\end{aligned}
$$

Substituting (9) - (11) and

$$
\begin{aligned}
& \overline{a_{31}}=-1.250 \\
& \overline{a_{32}}=-2.425
\end{aligned}
$$

into (18) - (20), we can obtain the weight parameters

$$
\begin{aligned}
& \overline{w_{1}}=0.004 \\
& \overline{w_{2}}=0.039 \\
& \overline{w_{3}}=0.957 .
\end{aligned}
$$

From the obtained weight parameters, it is possible to grasp the meaning of the rational solution of the optimal SPM motor design problem. It becomes obvious that the objective function $f_{3}$ is much more worthy than $f_{1}$ and $f_{2}$. That is, the cogging torque is more valuable than the copper and iron losses. In addition, the objective function with respect to the copper loss $f_{1}$ is much less worthy than that with respect to the iron loss $f_{2}$.

When the unit of the objective functions is the same, it is easy to compare the value of functions and to decide their weight parameter. However, for the objective functions with the different unit, the game theory can give the rational solution. It is hard to grasp the meaning of the rational solution of such a problem. The proposed method posteriori 


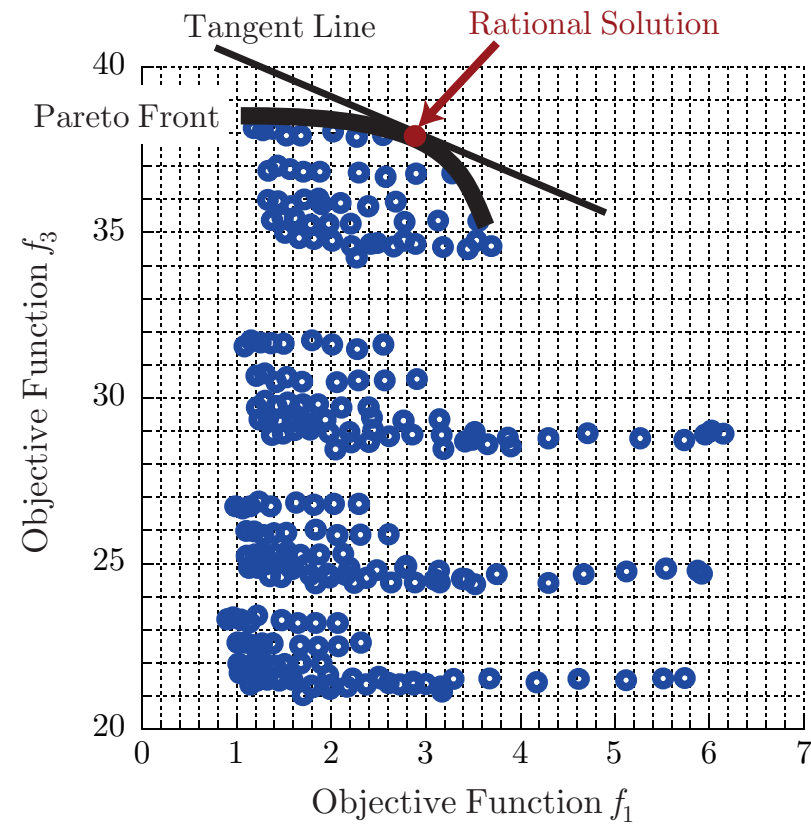

Fig. 5. The exploration points and the rational solution in the objective functions $f_{1}$ and $f_{3}$. The tangent line touches the Pareto front at the rational solution. The open circles are the exploration point of the game theory. The rational point is $\left(\overline{f_{1}}, \overline{f_{3}}\right)=(2.89,37.91)$, and the tangent line is $f_{1}=-1.250 f_{3}+41.532$.

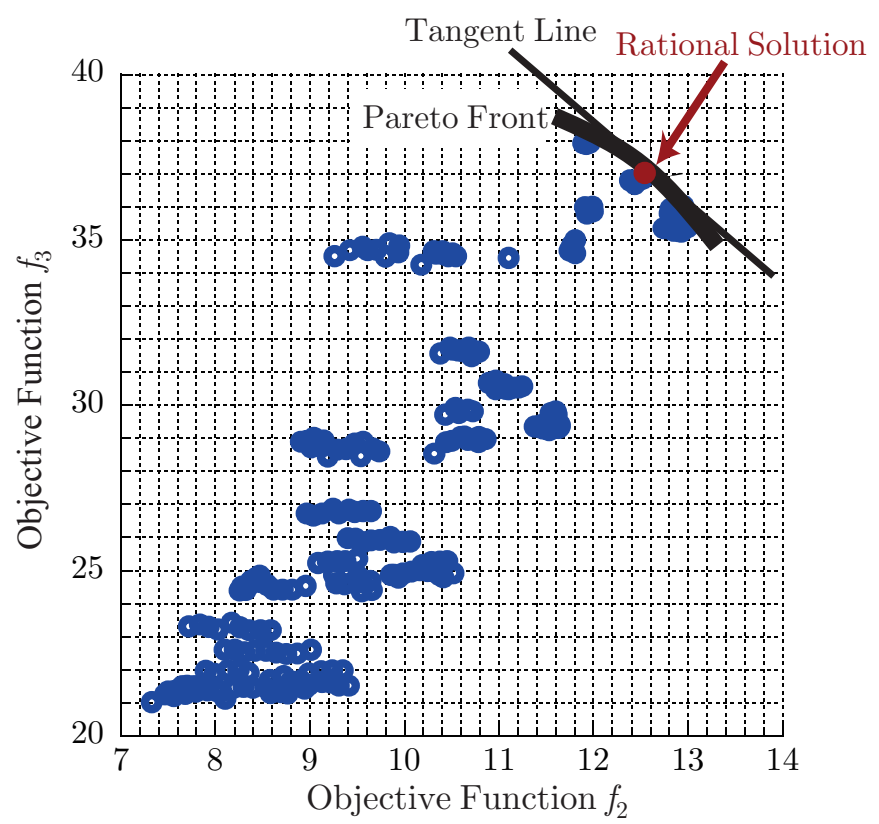

Fig. 6. The exploration points and the rational solution in the objective functions $f_{2}$ and $f_{3}$. The line touches the Pareto front at the rational solution. The open circles are the exploration point of the game theory. The rational point is $\left(\overline{f_{2}}, \overline{f_{3}}\right)=(11.89,37.91)$, and the tangent line is $f_{2}=-2.425 f_{3}+67.624$

interprets the meaning of the rational solution by means of calculating the weight parameters. The different values of weight parameters can be obtained in an optimization problem even with the same unit of the objective functions. In fact, in the SPM motor design problem, the iron loss is evaluated much more than the copper one, and the greatest worth is provided to the cogging torque.

\section{CONCLUSION}

We have previously proposed that the game theory is utilized for an optimal design problem of electromagnetic apparatuses even though the single objectives are conflicting and tradingoff. It was successful to obtain the rational solution based on the game theory. However, the meaning of the ration solution obtained was unclear. Therefore, we propose a method of clarifying the meaning of the rational solution as a post process. The proposed method gives the weight parameters of the weighted summed objective functions so that its optimal value is identical to the rational solution. We can intuit the meaning of the rational solution by comparing the weight parameters.

For examining the proposed method, it is applied to the optimal design problem of the SPM motor presented in [6]. Its objectives are to minimize the copper loss, the iron loss, and the cogging torque simultaneously. The obtained rational solution is evaluated from the calculated weight parameters that the cogging torque is the most important.

When employing the game theory, the small number of the design variables is considered as compared with the ordinary optimization method. In addition, the game theory deals with only discrete variable parameters. For wide exploring, the ordinary optimization method, such as genetic algorithm, simulated annealing, or particle swarm, should be employed to fully optimally design electromagnetic apparatuses after calculating the weight parameters by the proposed method.

\section{REFERENCES}

[1] K. Watanabe, F. Campelo, Y. Iijima, K. Kawano, T. Matsuo, T. Mifune, and H. Igarashi, "Optimization of inductors using evolutionary algorithms and its experimental validation," IEEE Trans. Magn., vol. 46, no. 8, pp. 3393-3396, Aug. 2010.

[2] J. A. Tapia, J. Pyrhone, J. Puranen, P. Lindh, and S. Nyman "Optimal design of large permanent magnet synchronous generators," IEEE Trans. Magn., vol. 49, no. 1 , pp. 642-650, Jan. 2013.

[3] J. Lee and B. Kwon, "Optimal rotor shape design of a concentrated flux IPM-type motor for improving efficiency and operation range," IEEE Trans. Magn., vol. 49, no. 5, pp. 2205-2208, May 2013.

[4] C. A. C. Coelle, G. T. Pulido, and M. S. Lechuga, "Handling multiple objectives with particle swarm optimization," IEEE Trans. Evolutionary computation, vol. 8, no. 3, pp. 256-279, 2004.

[5] C. Magele, A. Köstinger, M. Jaindl, W. Renhart, B. Cranganu-Cretu, and J. Smajic, "Niching evolution strategies for simultaneously finding global and Pareto optimal solutions," IEEE Trans. Magn., vol. 46, no. 8, pp. 2743-2746, Aug. 2010.

[6] T. Miyamoto, S. Noguchi, and H. Yamashita, "Selection of an optimal solution for multiobjective electromagnetic apparatus design based on game theory," IEEE Trans. on Magn., vol. 44, no. 6, pp. 1026-1029, Jun. 2008 .

[7] K. Binmore, Playing for Real-A Text on Game Theory. Oxford, U.K.: Oxford Univ. Press, 2007.

[8] M. J. Osvorne and A. Rubinstein, A Course in GameTheory. Cambridge, MA: MIT Press, 1994.

[9] M. Cioffi, P. Di Barba, A. Formisano, and R. Martone, "Pareto optima and Nash equilibria: An effective approach to the shape design in electromagnetics," COMPEL: The International Journal for Computation and Mathematics in Electrical and Electronic Engineering, vol. 27, no. 4, pp. 845-854, 2008.

[10] S. Matsutomo, S. Noguchi, H. Yamashita, and S. Tanimoto, "A new concept for optimal design method considering modeling accuracy of electromagnetic device," IEEE Trans. on Magn., vol. 40, no. 2, pp. 1232 1235, Feb. 2004. 